\title{
Matière quantique sous champ magnétique / Quantum matter under magnetic field
}

Institut de physique

\section{Benoît Fauqué}

\section{OpenEdition}

\section{Journals}

Édition électronique

URL : https://journals.openedition.org/annuaire-cdf/16202

DOI : 10.4000/annuaire-cdf.16202

ISBN : 978-2-7226-0572-5

ISSN : 2109-9227

\section{Éditeur}

Collège de France

\section{Édition imprimée}

Date de publication : 30 décembre 2020

Pagination : 677-678

ISBN : 978-2-7226-0516-9

ISSN : 0069-5580

\section{Référence électronique}

Benoît Fauqué, « Matière quantique sous champ magnétique / Quantum matter under magnetic field », L'annuaire du Collège de France [En ligne], 118 | 2020, mis en ligne le 01 avril 2021, consulté le 22 août 2022. URL : http://journals.openedition.org/annuaire-cdf/16202 ; DOI : https://doi.org/10.4000/ annuaire-cdf. 16202 


\title{
INSTITUT DE PHYSIQUE
}

\section{MATIÈRE QUANTIQUE SOUS CHAMP MAGNÉTIQUE / QUANTUM MATTER UNDER MAGNETIC FIELD}

\author{
Responsable : Benoît FAUQUÉ
}

\begin{abstract}
RECHERCHE
Page web : https://www.college-de-france.fr/site/young-team-incubator/ Presentation_4.htm.

Le groupe Matière quantique sous champ magnétique de l'IPCF s'intéresse aux propriétés électroniques des métaux dilués en présence d'un champ magnétique. Sous l'effet du champ magnétique, les électrons se déplacent le long d'une hélice dont le rayon est donné par le rayon cyclotronique (Rc). À mesure que le champ magnétique augmente, Rc diminue, croisant les différentes échelles caractéristiques du matériau. À faible champ magnétique, lorsque $\mathrm{r}_{\mathrm{c}}$ est comparable au libre parcours moyen électronique $\left(l_{e}\right)$, la dynamique de l'électron est bien décrite par une approche semi-classique. Â plus fort champ magnétique, lorsque Rc devient comparable à la longueur d'onde de Fermi $\left(\lambda_{\mathrm{F}}\right)$, on s'attend à une modification de l'état fondamental électronique du système. C'est dans ce régime dit de la «limite quantique » que l'effet Hall quantique est observé à deux dimensions. Paradoxalement, cette limite a été peu étudiée à trois dimensions, essentiellement pour une raison pratique : dans le cas du cuivre (ou d'un métal standard), le champ magnétique nécessaire pour atteindre cette limite est de l'ordre de 5000 T. En revanche, dans le cas des métaux dilués, celui-ci est beaucoup plus faible, de l'ordre de quelques teslas. Nous explorons donc l'organisation des électrons dans le régime de la limite quantique dans les métaux dilués en particulier dans le graphite où nous avons mis en évidence l'existence de phases électroniques originales induites par le champ magnétique.
\end{abstract}

\section{Publications}

Lin X., Rischau C.W., Buchauer L., JaOui A., Fauqué B. et Behnia K., « Metallicity without quasi-particles in room-temperature strontium titanate », NPJ Quantum Materials, vol. 2, no 1, 2017, p. 41, DOI : 10.1038/s41535-017-0044-5.

Collignon C., Fauqué B., Cavanna A., Gennser U., Mailly D. et Behnia K., "Superfluid density and carrier concentration across a superconducting dome: the case of $\mathrm{SrTi}_{1-\mathrm{x}} \mathrm{Nb}_{\mathrm{x}} \mathrm{O}_{3}$ », Physical Review B, vol. 96, no 22, 2017, 224506, DOI : 10.1103/ PhysRevB.96.224506 [arXiv: 1703.00863].

LeBoeuf D., Rischau C.W., Seyfarth G., Küchler R., Berben M., Wiedmann S., TABIS W., Frachet M., BeHNIA K. et FAUQUÉ B., « Thermodynamic signatures of the fieldinduced states of graphite », Nature Communications, vol. 8, $\mathrm{n}^{\circ}$ 1, 2017, 1337, DOI : 10.1038/ s41467-017-01394-7 [arXiv: 1705.07056]. 
ZHu Z., FAuqué B., BehNiA K. et FusEYA Y., « Magnetoresistance and valley degree of freedom in bulk bismuth », Journal of Physics: Condensed Matter, vol. 30, $\mathrm{n}^{\mathrm{O}}$ 31, 2018, 313001, DOI : 10.1088/1361-648X/aaced7 [arXiv: 1801.07098].

Collignon C., Lin X., Rischau C.W., Fauqué B. et Behnia K., « Metallicity and superconductivity in doped strontium titanate », Annual Review of Condensed Matter Physics, vol. $10, \mathrm{n}^{\mathrm{o}}$ 1, 2019, p. 25-44, DOI : 10.1146/annurev-conmatphys-031218-013144 [arXiv: 1804.07067].

\section{RECHERCHES SUR LA PHYSIQUE MÉSOSCOPIQUE À PARTIR DE GRAPHĖNE ET DE JONCTIONS JOSEPHSON / JOSEPHSON JUNCTION SPECTROSCOPY OF MESOSCOPIC SYSTEMS}

Responsable : Çağlar GiRiT

\section{RECHERCHE}

Page web : https://www.college-de-france.fr/site/young-team-incubator/ Presentation_2.htm.

\section{PHOTONIQUE QUANTIQUE / QUANTUM PHOTONICS}

Responsable : Alexei OURJOUMTSEV

\section{RECHERCHE}

Page web : https://www.college-de-france.fr/site/young-team-incubator/ Presentation_3.htm.

\section{PHYSIQUE QUANTIQUE}

Responsable : Jean-Michel RAIMOND

\section{RECHERCHE}

Page web : https://www.college-de-france.fr/site/physique-quantique/Presentation.htm.

Notre activité s'est orientée en 2017-2018 vers trois directions principales : jeux quantiques dans les multiplicités de Rydberg; électrodynamique quantique en cavité ; simulation quantique avec les atomes de Rydberg. 